\title{
LABS problem and ground state spin glasses system
}

\author{
A.N. Leukhin*, V.I. Bezrodnyi, and Yu.A. Kozlova \\ Mari State University, 424000 Yoshkar-Ola, Russia
}

\begin{abstract}
In our work we demonstrate the new results of an exhaustive search for optimal binary sequences with minimum peak sidelobe (MPS) up to length $\mathrm{N}=85$. The design problem for law autocorrelation binary sequences (LABS) is a notoriously difficult computational problem which is numbered as the problem number 005 in CSPLib. In statistical physics LABS problem can be interrepted as the energy of $\mathrm{N}$ iteracting Ising spins. This is a Bernasconi model. Due to this connection to physics we refer a binary sequence as one-dimensional spin lattice. At this assumption optimal binary sequences by merit factor (MF) criteria are the ground-state spin system without disorder which exhibits a glassy regime.
\end{abstract}

Design method for ground-state level quantum system for one-dimensional Ising spin chain [1] consisting from $\mathrm{N}$ mutual equal-distance particles is offered. Within the frame of Bernasconi model [2] the design problem of simplest quantum system presented like onedimensional Ising model is reduced to the design problem of binary sequences with lowest level of energy of sidelobes $[3,4]$ of aperiodic autocorrelation. In fact, the Bernasconi model exhibits features of a glass transition like a jump in the specific heat and slow dynammics and aging.

The problem of the glass state remains one major unsolved issued in condensed matter theory. Despite an enormous body of experimental and numerical data and quite detailed phenomenological theories, there is no fully stisfactory microsopic model for the glass state. Within the Bernascni model the high temperature phase of Ising spin system reproduces exactly an approximation due to Golay [3]. For the low-temperature regime, analytical results are rare - especialy for the ground state are not known. Low temperature glass phase is rather similar to the low temperature phase of Derrida's random energy model [15].

There are two criteria for the optimality of binary sequences with low levels of aperiodic autocorrelation: minmum peak sidlobe (PSL) and maximum MF. The effective computing methods for an exhaustive search of binary sequences with maximum MF are presented in [5-7]. Optimal binary sequences by MF criteria are consructed for the lengths $\mathrm{N}=[2,66]$. Enumerative algorihms (complete or partial) are limited to small values of $\mathrm{N}<300$ by the eponential complexity of local search algorithms. Analytical binary sequences for unlimited $\mathrm{N}$ with current record of an symptotic merit factor of $\mathrm{MF}=6.340261$ is set called appended rotated Legenfre sequences.

\footnotetext{
*Corresponding author: leukhinan@list.ru
} 
The effective computing method for an exhaustive search of MPS binary sequences is presented in [8]. Optimal binary sequences by MPS criteria are consructed for the lengths $\mathrm{N}=[2,85]$. The large values of $\mathrm{N}$ exhaustive enumeration is not feasible and one has to resort either partial enumerations or heuristic searches. In both cases one obtains sequences with small PSL but not necessarily minimum peaksidelobe. The good compilation of results for lengths $\mathrm{N}=[2 ; 300]$ and for some lengths between $\mathrm{N}=303$ and $\mathrm{N}=1000$ and for some lengths between $\mathrm{N}=1019$ and $\mathrm{N}=4096$ is given in [16]. The results on adiabatic quantum computer D-Wave 2 for finding low peak sidelobes codes with lengths more than 100 and up to 426 are presented in [9]. The theoretical bound for MPS sequences is offered in [17] and it is given as $P S L \approx 0.435 \sqrt{N}$. Some analytical constructions of binary sequences has a PSL between $0.9 \sqrt{N}<P S L<1.1 \sqrt{N}$.

We present as some improvements to previously known exhaustive search algorithm for MPS sequences and as some improvements to previously known results of an exhaustive search for MPS [10-13]. An exhaustive search of binary MPS sequences was performed off and on during 32 months using high performance computing grid [14]. Total performance of MarGrid is around 100T/Flops with 120 Intel Xeon E5-2679 v3 processors, with 100 Intel 17 Core processors and with supercomputer Flagman RX240 on the base of 8 NVIDIA TESLA C2059 with 3584 parallel graphical processors and on the base of 2 processors Intel Xeon X5670 (up to Six-Core). For example an exhaustive search for the length $N=80$ was performed in the background for 1 month. Our computational results are compared with results of some different stochastic local search methods for binary sequences such as: evolutionary algorithm, constrained local search, local search relaxation, direct stochastic search, tabu search, memetic, ants colony optimization, simulated annealing, genetic, iterative local search, scatter search. Executing an exhaustive search for binary sequenceswith $\mathrm{PSL}=4$ we proove that there are no exist any binary sequences with $\mathrm{PSL}=4$ with the lengths $\mathrm{N}=83,84,85$.

This work was supported by Russian Ministry of Education and Science (02.G25.31.0204, 2014/81/1856), Russian Foundation of Basic Research (15-07-99514).

\section{References}

1. E. Ising, Z. Phys. 31, 253 (1925)

2. J. Bernasconi, J. Phys. 48, 559 (1987)

3. M.J.E. Golay, IEEE Trans. Inf. Theory IT-28, 543 (1982)

4. CSPLib: a problem library for contraints (www.cslib.org)

5. S. Mertens, J. Phys A: Math. 29, 473 (1996)

6. H. Bauke, S. Mertens, Phys. Rev. E 70, 025102 (2004)

7. T. Packebusch, S. Mertens, J.Phys. A: Math. Theory. 49, 165001 (2016)

8. G.E. Coxson, J.C. Russo, IEEE Trans. on Aerosp. and El. Syst. 41, 302 (2005)

9. G.E. Coxson, C.R. Hill, J.C. Russo, HPEC Conf. 7 (2014)

10. A.N. Leukhin, E.N. Potekhin., Proc. EuRad. 495 (2013)

11. A.N. Leukhin, A.S. Shuvalov, E.N. Potekhin, Bull. RAS. Phys. 78, 207 (2014)

12. A.N. Leukhin, E.N. Potekhin, Lect. Notes in Comp. Sci. 8865, 157 (2014)

13. A.N. Leukhin, E.N. Potekhin, Journal of Phys. Conf. Ser. 613, 012006 (2015)

14. hpc.marsu.ru

15. B. Derrida, Phys. Rev. B 24, 2613 (1981)

16. W.H. Mow, K.L. Du, W.H. Wu, IEEE Trans. on Aerosp. and El. 51, 290 (2015)

17. L. Ein-Dor, I. Kanter, W. Kinzel, Phys. Rev. E 65, 020102 (2002) 